\title{
MIMO Radar Detection in non-Gaussian and
}

\section{Heterogeneous Clutter}

\author{
Chin Yuan Chong, Member, IEEE, Frédéric Pascal Member, IEEE,, \\ Jean-Philippe Ovarlez Member, IEEE, and Marc Lesturgie Member, IEEE,
}

\begin{abstract}
In this paper, the Generalized Likelihood Ratio Test - Linear Quadratic (GLRT-LQ) has been extended to the Multiple-Input Multiple-Output (MIMO) case where all transmit-receive subarrays are considered jointly as a system such that only one detection threshold is used. The GLRT-LQ detector has been derived based on the Spherically Invariant Random Vector (SIRV) model and is Constant False Alarm Rate (CFAR) with respect to the clutter power fluctuations (also known as the texture). The new MIMO detector is then shown to be texture-CFAR as well. The theoretical performance of this new detector is first analytically derived and then validated using Monte-Carlo simulations. Its detection performance is then compared to that of the well-known Optimum Gaussian Detector (OGD) under Gaussian and non-Gaussian clutter. Next, the adaptive version of the detector is investigated. The covariance matrix is estimated using the Fixed Point (FP) algorithm which enables the detector to remain texture- and matrix-CFAR. The effects of the estimation of the covariance matrix on the detection performance are also investigated.
\end{abstract}

\section{Index Terms}

MIMO Radar, Non-Gaussian Clutter, SIRV, Detection Performance, GLRT-LQ

C. Y Chong is with SONDRA, Supélec, 3 rue Joliot-Curie, 91190 Gif-sur-Yvette, France and also with DSO National Laboratories, 20 Science Park Drive, Singapore 118230 (e-mail: chinyuan.chong@ supelec.fr)

F. Pascal is with SONDRA, Supélec, 3 rue Joliot-Curie, 91190 Gif-sur-Yvette, France (e-mail: frederic.pascal@ supelec.fr)

J-P. Ovarlez is with ONERA-DEMR, Chemin de la Hunière, 91761 Palaiseau Cedex, France and also with SONDRA, Supélec, 3 rue Joliot-Curie, 91190 Gif-sur-Yvette, France (e-mail: jean-philippe.ovarlez@onera.fr)

M. Lesturgie is with SONDRA, Supélec, 3 rue Joliot-Curie, 91190 Gif-sur-Yvette, France and also with ONERA-DEMR, Chemin de la Hunière, 91761 Palaiseau Cedex, France (e-mail: marc.lesturgie@ supelec.fr) 


\section{INTRODUCTION}

Multiple-Input Multiple-Output (MIMO) is a technique used in communications to increase data throughput and link range without additional bandwidth or transmit power. This is achieved by higher spectral efficiency and link reliability or diversity. Recently, this concept has been used for radar applications [1]. In the context of radar, a (statistical) MIMO radar is one where both the transmit and receive elements are sufficiently separated so as to provide spatial diversity. This reduces the fluctuations of the target Radar Cross Section (RCS) due to the different target aspects seen by each pair of transmitreceive elements [2]. It can also be used to improve the probability of detection [3] and resolutions [4]. Several applications including target classification and high-resolution imaging can be enhanced by a MIMO radar. On top of that, each transmit element sends a different (orthogonal) waveform which can be separated at the receive end. This provides waveform diversity which in turn increases the separation between clutter and target returns [5]. According to [6], it also improves the identifiability of target parameters, enables the direct application of adaptive arrays for target detection and enhances flexibility for transmit beampattern design.

MIMO procedures for radar have been widely studied for the case of additive Gaussian noise. In [3], [7], [8], the authors consider the detection performance for widely separated antennas while in [6], [9] the authors consider colocated antennas. The combined case of having widely separated antenna subarrays which contain colocated antenna elements is considered in [10]. However, these detectors may exhibit poor performance when the additive noise is no longer Gaussian.

In the case of MIMO radar, it is even more important to consider non-Gaussian models. Firstly, as mentioned earlier, one advantage of a MIMO imaging radar is improved resolution. Usually, in each resolution cell, there is a large number of scatterers. According to the Central Limit Theorem (CLT), the clutter power in each cell is almost constant and the clutter is considered to follow a Gaussian distribution. However, as the resolution cell becomes smaller, there are fewer scatterers in it and the CLT will no longer apply. Non-Gaussian models which take into account the variation in clutter power therefore have to be used. Moreover, as the resolution cell becomes smaller, it is more likely for the illuminated area to be non-homogeneous.

Secondly, for configurations with widely separated transmit-receive sub-systems, the target returns received by each receive sub-system are different due to different aspect angles, thus reducing target RCS fluctuations. Similarly, the clutter returns vary from sub-system to sub-system. Hence, it is important to use non-Gaussian models which better reflect the clutter power fluctuations. Indeed, experimental radar 
clutter measurements [11], [12], [13], [14] have been found to fit non-Gaussian statistical models such as the well-known Spherically Invariant Random Vector (SIRV) model [15], [16], [17] which has been widely studied, particularly in terms of detection [18], [19], [20].

In this paper we consider MIMO radar detection under non-Gaussian heterogeneous and impulsive clutter. The non-Gaussian statistical model we use is the SIRV model. This model writes clutter returns as the product of a Gaussian random process (speckle) and the square-root of a non-negative random variable $\tau$ (texture). Speckle models temporal fluctuations of clutter while texture models spatial fluctuations of clutter power. The SIRV can model different non-Gaussian clutter depending on the chosen texture, with Gaussian clutter as the special case where the texture is a constant. It also has a Gaussian kernel which means that certain classical results can still be applied. For example, the Maximum Likelihood (ML) estimates of target parameters are given by the maximization of the traditional matched filter.

In radar applications, the clutter covariance matrix is usually unknown and has to be estimated from target-free secondary data. Under non-Gaussian clutter, the classical Sample Covariance Matrix (SCM) is no longer the ML estimate. Hence, we consider here the Fixed Point Estimate (FPE) which was first introduced in [21], [22] and then fully analyzed in [23], [24]. FPE is the exact ML estimate when the texture is assumed to be deterministic and unknown. On the other hand, when $\tau$ is a positive random variable, FPE is an approximate ML estimate.

The main contribution of this paper is the derivation of a MIMO non-Gaussian detector for Constant False Alarm Rate (CFAR) detection and estimation. Previously, several Generalized Likelihood Ratio Tests (GLRTs) like the GLRT-Linear Quadratic (GLRT-LQ) detector in [18], [19] and asymptotic Bayesian Optimum Radar Detector (aBORD) in [20] have been derived based on the SIRV model and have been shown to be CFAR with respect to the texture (texture-CFAR). We generalized the GLRT-LQ detector to the MIMO case where all subarrays are considered jointly as a system such that only one detection threshold is used to regulate the false alarm rate. The resulting MIMO detector is also texture-CFAR. The Gaussian case is a special case of SIRV where the Probability Density Function of the texture is given by $p(\tau)=\sigma^{2} \delta(\tau-1)$ and $\sigma^{2}$ is the clutter power. Hence, this new detector is expected to give similar results as the well-known Optimum Gaussian Detector (OGD) under Gaussian clutter and superior results under non-Gaussian clutter.

The adaptive version of this new MIMO detector is then considered. Using the FPE to estimate the covariance matrix, this detector is shown to be texture-CFAR. Moreover, it is matrix-CFAR as it does not depend on the unknown covariance matrix. The detection performance depends on an additional parameter, $L_{r}$ : the number of secondary data containing only clutter returns which are used to obtain the 
FPE.

This paper is organized as follows. Firstly, we consider a general signal model for MIMO radar (Section II). Instead of considering a single element at each location, we assume that there is a subarray containing one or more elements. Section III-A shows the derivation of a MIMO non-Gaussian detector. In Section III-B, the theoretical performance of this new detector is derived and verified using Monte-Carlo simulations. The detection performance is then analyzed through Monte-Carlo simulations and compared to that of the OGD (extended to the MIMO case in [7], [25]) under both Gaussian and non-Gaussian clutter (Section III-C). Next, in Section IV-A, the adaptive version of this new detector is considered, using the FPE as the covariance matrix estimate. The effects of the covariance matrix estimation on the detection performance are investigated using Monte-Carlo simulations in Section IV-B. Finally, conclusions are presented in Section V.

\section{Signal Model}

In this article vectors and matrices are denoted by lower-case and upper-case bold, respectively. The superscript " $\nmid$ " denotes the Hermitian operator.

In this section, we consider a target located at $(x, y)$. Let there be $\tilde{N}$ transmit subarrays and $\tilde{M}$ receive subarrays. The $n$-th transmit and $m$-th receive subarray contain $N_{n}$ and $M_{m}$ elements respectively, for $n=1, \ldots, \tilde{N}$ and $m=1, \ldots, \tilde{M}$. The configuration can be seen in Fig. 1 . The RCS of the target seen by each receive-transmit pair is assumed to be different but deterministic. The elements within each subarray are assumed to be closely-spaced such that the RCS is the same.

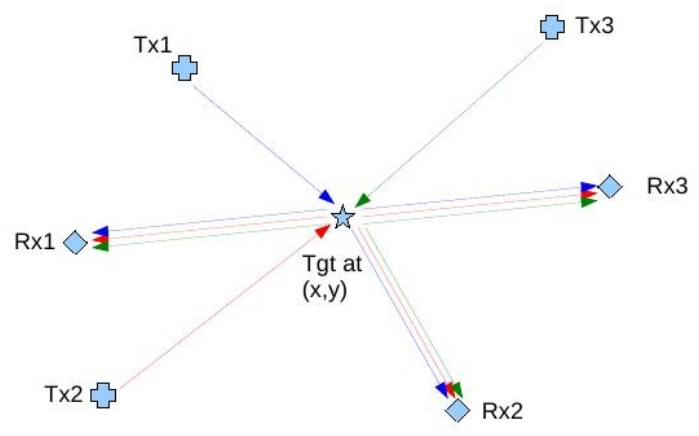

Fig. 1. The configuration where $\tilde{N}=\tilde{M}=3$.

Let $\mathbf{v}\left(\theta_{t, n}\right)$ and $\mathbf{a}\left(\theta_{r, m}\right)$ be the steering vectors and $\theta_{t, n}$ and $\theta_{r, m}$ the angular location for the target for the $n$-th transmit and $m$-th receive subarray respectively. Assuming that orthogonal waveforms are 
transmitted, the received signal after matched filtering can be expressed as:

$$
\begin{aligned}
\mathbf{y}_{m, n}\left(\tau_{m, n}\right) & =\mathrm{B}(m, n) \mathbf{a}\left(\theta_{r, m}\right) \otimes \mathbf{v}\left(\theta_{t, n}\right)+\mathbf{z}_{m, n} \\
& =\mathrm{B}(m, n) \mathbf{p}_{m, n}+\mathbf{z}_{m, n}
\end{aligned}
$$

where $\tau_{m, n}$ is the corresponding time-delay for $m-n$ receive-transmit pair and $\mathbf{B}$ is the $\tilde{M} \times \tilde{N}$ matrix containing the RCS of the target seen by each receive-transmit pair. The vector $\mathbf{p}_{m, n}$ is the $M_{m} N_{n} \mathrm{x} 1$ bistatic angular steering vector which is equal to $\mathbf{a}\left(\theta_{r, m}\right) \otimes \mathbf{v}\left(\theta_{t, n}\right)$ and $\otimes$ stands for the Kronecker product.

The vector $\mathbf{z}_{m, n}$ is a $M_{m} N_{n} \mathrm{x} 1$ vector containing the clutter returns and it is modeled by SIRV which is essentially a non-homogeneous and non-Gaussian process with random power. The variation in the power arises from the spatial variation in the backscattering of the clutter. According to [16], a SIRV is the product of the square root of a positive random variable $\tau$ (texture) and a $L$-dimensional independent complex circular Gaussian vector $\mathbf{x}$ :

$$
\mathbf{z}=\sqrt{\tau} \mathbf{x}
$$

$\mathbf{x}$ has zero mean and covariance matrix $\mathbf{M}$, denoted by $\mathcal{C N}(\mathbf{0}, \mathbf{M}) . \mathbf{M}=\mathbb{E}\left[\mathbf{x x}^{\dagger}\right]$ is assumed to be normalized such that $\operatorname{tr}(\mathbf{M})=L$ for identifiability considerations [21]. $\mathbb{E}[\cdot]$ denotes the expectation and $\operatorname{tr}(\cdot)$ the trace. The PDF of a SIRV is then given by:

$$
p_{\mathbf{z}}(\mathbf{z})=\int_{0}^{\infty} g_{\mathbf{z}}(\mathbf{z}, \tau) p(\tau) d \tau
$$

where $p(\tau)$ is the texture PDF and

$$
g_{\mathbf{z}}(\mathbf{z}, \tau)=\frac{1}{(\pi \tau)^{L}|\mathbf{M}|} \exp \left(-\frac{\mathbf{z}^{\dagger} \mathbf{M}^{-1} \mathbf{z}}{\tau}\right) .
$$

For each radar parameter to be determined, a steering vector is required. The resulting steering vector is simply the Kronecker product of all the steering vectors. In the bistatic case, as the transmit and receive angles are no longer the same, a separate steering vector is required for transmit $\left(\mathbf{v}\left(\theta_{t, n}\right)\right)$ and receive $\left(\mathbf{a}\left(\theta_{r, m}\right)\right)$.

For simplicity of notation, $\mathbf{y}_{m, n}\left(\tau_{m, n}\right)$ will be written simply as $\mathbf{y}_{m, n}$.

\section{MIMO NON-GAUSSIAN DETECTOR}

\section{A. Derivation}

Let us now consider the detection problem as the following binary hypothesis test:

$$
\left\{\begin{array}{ll}
\mathrm{H}_{0}: & \mathbf{y}_{m, n}=\mathbf{z}_{m, n} \\
\mathrm{H}_{1}: & \mathbf{y}_{m, n}=\mathrm{B}(m, n) \mathbf{p}_{m, n}+\mathbf{z}_{m, n}
\end{array} \quad n=1, \ldots, \tilde{N}, m=1, \ldots, \tilde{M}\right.
$$


Under the hypothesis $\mathrm{H}_{0}$, it is assumed that the received signal contains only clutter returns and hence there is no target. Under the hypothesis $\mathrm{H}_{1}$, it is assumed that the received signal contains a deterministic signal on top of the clutter returns and hence a target is present at the location $(x, y)$.

The dimension of each received signal, $M_{m} N_{n}$, is assumed to be greater than one since the steering vector does not give any information if its length is one.

a) $\tilde{M}=1$ and $\tilde{N}=1$

We begin with the case where $\tilde{M}=1$ and $\tilde{N}=1$. The dimension of the received signal is $L=M_{1} N_{1}$.

As there is only one received signal and steering vector, let them be denoted simply by $\mathbf{y}=\mathbf{y}_{1,1}$ and $\mathbf{p}=\mathbf{p}_{1,1}$. The classical likelihood ratio test is given by:

$$
\Lambda(\mathbf{y})=\frac{p\left(\mathbf{y} \mid \mathrm{H}_{1}\right)}{p\left(\mathbf{y} \mid \mathrm{H}_{0}\right)} \underset{\mathrm{H}_{0}}{\stackrel{\mathrm{H}_{1}}{\gtrless}} \eta .
$$

If we assume that the covariance matrix $\mathbf{M}$ is known and according to [20], $p\left(\mathbf{y} \mid \mathrm{H}_{1}\right)$ and $p\left(\mathbf{y} \mid \mathrm{H}_{0}\right)$ are replaced by their Bayesian estimates and asymptotically, we obtain:

$$
\Lambda_{1}(\mathbf{y})^{L}=\left[\frac{1}{1-\frac{\left|\mathbf{p}^{\dagger} \mathbf{M}^{-1} \mathbf{y}\right|^{2}}{\left(\mathbf{p}^{\dagger} \mathbf{M}^{-1} \mathbf{p}\right)\left(\mathbf{y}^{\dagger} \mathbf{M}^{-1} \mathbf{y}\right)}}\right]^{L} \stackrel{\mathrm{H}_{0}}{\underset{\mathrm{H}_{1}}{\gtrless}} \eta,
$$

which is equivalent to:

$$
1-\frac{1}{\Lambda_{1}(\mathbf{y})}=\frac{\left|\mathbf{p}^{\dagger} \mathbf{M}^{-1} \mathbf{y}\right|^{2}}{\left(\mathbf{p}^{\dagger} \mathbf{M}^{-1} \mathbf{p}\right)\left(\mathbf{y}^{\dagger} \mathbf{M}^{-1} \mathbf{y}\right)} \underset{\mathrm{H}_{0}}{\gtrless} \frac{\sqrt[L]{\eta}-1}{\sqrt[L]{\eta}},
$$

and leads to the GLRT-LQ test. The same test is derived in [18] through an asymptotic development of the test statistic in the GLRT and in [19] it is based on the ML estimation of the clutter power. In [18], the probability of false alarm of the test is given by $P_{f a}=(\sqrt[L]{\eta})^{-L+1}$. This expression gives us a $P_{f a}$ of one when $L=1$. This implies that the test breaks down as more than one element is required to estimate the clutter power. Note that no explicit form on the steering vector is assumed in the derivation of the GLRT-LQ. Thus, these detectors can also be applied to our case where $\mathbf{p}=\mathbf{a}\left(\theta_{r, 1}\right) \otimes \mathbf{v}\left(\theta_{t, 1}\right)$.

b) $\tilde{M} \geq 1$ and $\tilde{N} \geq 1$

We consider now the general case where $\tilde{M} \geq 1$ and $\tilde{N} \geq 1$. As the transmit-receive subarrays are widely separated, the clutter returns can be considered to be independent, hence $\mathbf{z}_{m, n}$ are independent 
and the likelihood ratio test becomes:

$$
\begin{aligned}
& \Lambda(\mathbf{Y})=\frac{p\left(\mathbf{Y} \mid \mathrm{H}_{1}\right)}{p\left(\mathbf{Y} \mid \mathrm{H}_{0}\right)}, \\
&=\frac{\prod_{m, n} p\left(\mathbf{y}_{m, n} \mid \mathrm{H}_{1}\right)}{\prod_{m, n} p\left(\mathbf{y}_{m, n} \mid \mathrm{H}_{0}\right)}, \\
&=\prod_{m, n} \frac{p\left(\mathbf{y}_{m, n} \mid \mathrm{H}_{1}\right)}{p\left(\mathbf{y}_{m, n} \mid \mathrm{H}_{0}\right)} \\
& \stackrel{\mathrm{H}_{0}}{\gtrless} \eta,
\end{aligned}
$$

where $\mathbf{Y}$ is the matrix containing all the received signals $\mathbf{Y}=\left[\mathbf{y}_{1,1}, \cdots, \mathbf{y}_{\tilde{M}, \tilde{N}}\right]$.

Using Eqn. (2), the GLRT-LQ, extended to the MIMO case, is given by:

$$
\Lambda(\mathbf{Y})=\prod_{m, n}\left[\frac{1}{1-\frac{\left|\mathbf{p}_{m, n}^{\dagger} \mathbf{M}_{m, n}^{-1} \mathbf{y}_{m, n}\right|^{2}}{\left(\mathbf{p}_{m, n}^{\dagger} \mathbf{M}_{m, n}^{-1} \mathbf{p}_{m, n}\right)\left(\mathbf{y}_{m, n}^{\dagger} \mathbf{M}_{m, n}^{-1} \mathbf{y}_{m, n}\right)}}\right]^{M_{m} N_{n}} \stackrel{\mathrm{H}_{0}}{\stackrel{\mathrm{H}_{1}}{\gtrless}} \eta,
$$

where $\mathbf{M}_{m, n}$ is the covariance matrix for the $m-n$ receive-transmit pair.

From Eqn. (4), we see that when the number of elements in each subarray, $M_{m} N_{n}$, is not a constant, the expression for MIMO GLRT-LQ is very complicated and it is difficult for its detection performance to be analyzed theoretically. Monte-Carlo simulations have to be run in such cases to obtain the detection performance. Instead, in the next section, we will consider the particular case where $M_{m} N_{n}=L$ is a constant.

c) $\tilde{M} \geq 1, \tilde{N} \geq 1$ and $M_{m} N_{n}=L$

If we consider now that the number of elements in each transmit and receive subarray is the same, i.e.

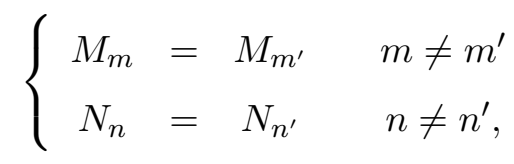

then the total number of elements in each subarray is the same:

$$
M_{m} N_{n}=L \quad \forall m=1, \ldots, \tilde{M}, n=1, \ldots, \tilde{N} .
$$

Moreover, as the returns from each transmit-receive pair are independent, having $\tilde{N}$ transmit subarrays and $\tilde{M}$ receive subarrays is equivalent to having one transmit subarray and $K=\tilde{N} \tilde{M}$ receive subarrays. Eqn. (4) can thus be simplified to be:

$$
\prod_{i=1}^{K} \Lambda_{i}\left(\mathbf{y}_{i}\right) \underset{\mathrm{H}_{0}}{\stackrel{\mathrm{H}_{1}}{\gtrless}} \sqrt[L]{\eta}=\lambda,
$$


where

$$
\Lambda_{i}\left(\mathbf{y}_{i}\right)=\left[\frac{1}{1-\frac{\left|\mathbf{p}_{i}^{\dagger} \mathbf{M}_{i}^{-1} \mathbf{y}_{i}\right|^{2}}{\left(\mathbf{p}_{i}^{\dagger} \mathbf{M}_{i}^{-1} \mathbf{p}_{i}\right)\left(\mathbf{y}_{i}^{\dagger} \mathbf{M}_{i}^{-1} \mathbf{y}_{i}\right)}}\right],
$$

and $\mathbf{M}_{i}$ is the covariance matrix of $\mathbf{y}_{i}$.

\section{B. Theoretical Performance}

The probability of false alarm $P_{f a}$ is the probability of choosing $\mathrm{H}_{1}$ when the target is absent $\left(\mathrm{H}_{0}\right)$ :

$$
P_{f a}=P\left(\prod_{i=1}^{K} \Lambda_{i}\left(\mathbf{y}_{i}\right)>\lambda \mid \mathrm{H}_{0}\right) .
$$

\section{Theorem III.1}

Given a MIMO radar system containing $K$ sub-systems and $L$ elements $(L>1)$ in each sub-system and using the detector given in Eqn. (5), the probability of false alarm is given by:

$$
P_{f a}=\lambda^{-L+1} \sum_{k=0}^{K-1} \frac{(L-1)^{k}}{k !}(\ln \lambda)^{k} .
$$

\section{Remark III.1}

- $P_{f a}$ depends only on $K$ and $L$ and not on the clutter parameters, hence showing the texture-CFAR property of the test given by Eqn. (5). Moreover, it is clear that the detector does not depend on the covariance matrices which can be different for each transmit-receive subarray.

- The closed-form expression is useful for the analysis of detection performance. Using Eqn. (7), the threshold to be used in the detection test is theoretically set to ensure a given $P_{f a}$.

Proof:

Under $\mathrm{H}_{0}$, the received signal contains only clutter returns:

$$
\mathbf{y}_{i}=\mathbf{z}_{i}=\sqrt{\tau_{i}} \mathbf{x}_{i}
$$

where $\mathbf{x}_{i} \sim \mathcal{C N}\left(\mathbf{0}, \mathbf{M}_{i}\right)$ and $\sim$ means to be distributed as. As $\tau_{i}$ is cancelled out in Eqn. (6), $\Lambda_{i}\left(\mathbf{y}_{i}\right)=$ $\Lambda_{i}\left(\mathbf{x}_{i}\right)$.

According to [26], the GLRT-LQ detector can be expressed in terms of an $F$-statistics:

$$
\frac{\left|\mathbf{p}_{i}^{\dagger} \mathbf{M}_{i}^{-1} \mathbf{y}_{i}\right|^{2}}{\left(\mathbf{p}_{i}^{\dagger} \mathbf{M}_{i}^{-1} \mathbf{p}_{i}\right)\left(\mathbf{y}_{i}^{\dagger} \mathbf{M}_{i}^{-1} \mathbf{y}_{i}\right)}=\frac{t}{t+1}
$$

where $(L-1) t$ is a centralized F-distributed random variable with parameters $v_{1}=2$ and $v_{2}=2 L-2$. The PDF of $t^{\prime}=(L-1) t$ can be expressed as:

$$
f\left(t^{\prime}\right)=\frac{\Gamma\left(\frac{v_{1}+v_{2}}{2}\right)}{\Gamma\left(\frac{v_{1}}{2}\right) \Gamma\left(\frac{v_{2}}{2}\right)} \frac{1}{t^{\prime}}\left(\frac{v_{1} t^{\prime}}{v_{1} t^{\prime}+v_{2}}\right)^{\frac{v_{1}}{2}}\left(1-\frac{v_{1} t^{\prime}}{v_{1} t^{\prime}+v_{2}}\right)^{\frac{v_{2}}{2}},
$$


where $\Gamma$ is the Gamma function as defined in [27]. As $v_{1}$ and $v_{2}$ are both positive integers, Eqn. (8) can be simplied to:

$$
f\left(t^{\prime}\right)=\left(\frac{L-1}{t^{\prime}+L-1}\right)^{L}
$$

Each $\Lambda_{i}\left(\mathbf{x}_{i}\right)$ can also be expressed in terms of $t^{\prime}$ :

$$
\Lambda_{i}\left(\mathbf{x}_{i}\right)=\frac{1}{1-\frac{t}{t+1}}=1+t=1+\frac{t^{\prime}}{L-1} .
$$

Let $u_{i}=\Lambda_{i}\left(\mathbf{x}_{i}\right)$ such that $t^{\prime}=\left(u_{i}-1\right)(L-1)$,

$$
\begin{aligned}
f\left(u_{i}\right) & =f\left(t^{\prime}\right) \cdot\left|\frac{d t^{\prime}}{d u_{i}}\right| \\
& =(L-1) u_{i}^{-L} .
\end{aligned}
$$

To find the PDF for $u=\prod_{i=1}^{K} u_{i}$, we consider the bijective function from $\left(u_{1}, \ldots, u_{K}\right)$ to $\left(u, v_{1}, \ldots, v_{K-1}\right)$ where:

$$
\begin{aligned}
u & =\prod_{i=1}^{K} u_{i} \in[1,+\infty), \\
v_{1} & =u_{1} \in[1, u], \\
v_{i} & =u_{i} \in\left[1, v_{i-1}\right] \quad i=2, \ldots, K-1 .
\end{aligned}
$$

To obtain the PDF of $u$, we perform a change of variable:

$$
\begin{aligned}
f_{1}\left(u_{1}, \ldots, u_{K}\right) & =\prod_{i}^{K} f\left(u_{i}\right)=(L-1)^{K} u^{-L}, \\
f_{2}\left(u, v_{1}, \ldots, v_{K-1}\right) & =\mathbb{1}_{u \geq v_{1} \geq \cdots \geq u_{K-1}} f_{1}\left(u_{1}, \ldots, u_{K}\right)|J|^{-1}=(L-1)^{K} u^{-L} \frac{1}{\prod_{i=1}^{K-1} v_{i}}, \\
f_{3}\left(u, v_{1}, \ldots, v_{K-2}\right) & =\mathbb{1}_{u \geq v_{1} \geq \cdots \geq u_{K-2}} \int_{1}^{v_{K-2}} f_{2}\left(u, v_{1}, \ldots, v_{K-1}\right) d v_{K-1}=(L-1)^{K} u^{-L} \frac{\ln v_{K-2}}{\prod_{i=1}^{K-2} v_{i}}, \\
f_{4}\left(u, v_{1}, \ldots, v_{K-3}\right) & =\mathbb{1}_{u \geq v_{1} \geq \cdots \geq u_{K-3}} \int_{1}^{v_{K-3}} f_{3}\left(u, v_{1}, \ldots, v_{K-2}\right) d v_{K-2}=(L-1)^{K} u^{-L} \frac{1}{2} \frac{\left(\ln v_{K-3}\right)^{2}}{\prod_{i=1}^{K-3} v_{i}}, \\
& \vdots \\
f_{K}(u) & =\int_{1}^{u} f_{K-1}\left(u, v_{1}\right) d v_{1}=(L-1)^{K} u^{-L} \frac{1}{(K-1) !}(\ln u)^{K-1} .
\end{aligned}
$$

To obtain the $P_{f a}$, we integrate $f_{K}(u)$ from the threshold $\lambda$ to infinity:

$$
\begin{aligned}
P_{f a} & =\int_{\lambda}^{\infty} f_{K}(u) d u, \\
& =\lambda^{-L+1} \sum_{k=0}^{K-1} \frac{(L-1)^{k}}{k !}(\ln \lambda)^{k} .
\end{aligned}
$$


To verify Theorem III.1, Monte-Carlo simulations $\left(N_{r}=10^{6}\right)$ are carried out. The parameters used can be seen in Table I.

\begin{tabular}{|c|c|c|c|c|c|}
\hline$\tilde{M}$ & $\tilde{N}$ & $M_{m}$ & $N_{n}$ & $K=\tilde{M} \tilde{N}$ & $L=M_{m} N_{n}$ \\
\hline 3 & 2 & 4 & 3 & 6 & 12 \\
\hline
\end{tabular}

TABLE I

PARAMEters used For Monte-CARlo Simulations.

Due to Remark III.1, the covariance matrix $\mathbf{M}_{i}$ of each $\mathbf{y}_{i}$, without loss of generalities, is chosen identically and equal to M. $\mathbf{M}$ is spatially colored and its elements are given by:

$$
\mathrm{M}(p, q)=\rho^{|p-q|} e^{j \frac{\pi}{2}(p-q)} .
$$

$\rho$ is the correlation coefficient and it is chosen to be 0.2 such that there is a slight correlation between different elements of the subarray.

Experimental radar clutter measurements have shown that the texture can be distributed according to a Gamma distribution [12], [13] or a Weibull one [28], [29]. When the texture is Gamma-distributed, the resulting clutter corresponds to one that is K-distributed. This has been widely studied in the litterature, see e.g. [19], [30], [31].

The PDF and the statistical mean of the Gamma distribution with parameters $a$ and $b$ are given by:

$$
\begin{aligned}
p(\tau) & =\frac{1}{b^{a} \Gamma(a)} \tau^{a-1} e^{-\tau / b}, \quad \Gamma(z)=\int_{0}^{\infty} t^{z-1} e^{-t} d t \\
\mathbb{E}[\tau] & =a b .
\end{aligned}
$$

The PDF and the statistical mean of the Weibull distribution on non-negative reals with parameters $a$ and $b$ are given by:

$$
\begin{aligned}
& p(\tau)=\frac{b}{a}\left(\frac{\tau}{a}\right)^{b-1} e^{-\left(\frac{\tau}{a}\right)^{b}}, \\
& \mathbb{E}[\tau]=a \Gamma\left(1+\frac{1}{b}\right) .
\end{aligned}
$$

The clutter power for each element, $\sigma^{2}$, is given by:

$$
\sigma^{2}=\mathbb{E}[\tau]
$$


In order to keep $\sigma^{2}$ constant, the two parameters of the distributions are set such that the statistical mean of the texture, given in Eqn. (10) and (12), remains the same. In this simulation, $\sigma^{2}$ is chosen to be one. The parameters used to simulate the texture are shown in Table II. The parameters are chosen such that for each texture, the first case is an instance of impulsive clutter while the second one is more similar to the Gaussian case.

For comparison, the Gaussian case where $p(\tau)=\sigma^{2} \delta(\tau-1)$, is also simulated. In this case, $\sigma^{2}$ is also equal to one.

\begin{tabular}{|c|c|c|c|}
\hline & Texture distribution & $a$ & $b$ \\
\hline 1 & Gamma & 0.5 & $\frac{\sigma^{2}}{a}=2$ \\
2 & Gamma & 2 & $\frac{\sigma^{2}}{a}=0.5$ \\
\hline 1 & Weibull & $\frac{\sigma^{2}}{\Gamma\left(1+\frac{1}{b}\right)}=0.7418$ & 0.658 \\
2 & Weibull & $\frac{\sigma^{2}}{\Gamma\left(1+\frac{1}{b}\right)}=1.1233$ & 1.763 \\
\hline
\end{tabular}

TABLE II

TEXTURE PARAMETERS USED FOR MONTE-CARLO SIMUlations.

On Fig. 2, we have plotted the " $P_{f a}$-threshold" curves under different hypotheses of the clutter distribution: Gaussian, K-distributed and Weibull-distributed texture. We see that there is perfect agreement between the theory given by Theorem III.1 and the simulation. The texture-CFAR property can also be seen clearly since the curves do not depend on the distribution of the texture.

\section{Simulation Results}

The probability of detection $P_{d}$ is the probability of correctly choosing $\mathrm{H}_{1}$ when the target is present:

$$
P_{d}=P\left(\prod_{i=1}^{K} \Lambda_{i}\left(\mathbf{y}_{i}\right)>\lambda \mid \mathrm{H}_{1}\right) .
$$

Under $\mathrm{H}_{1}$, the received signal contains both a deterministic signal and clutter returns:

$$
\mathbf{y}_{m, n}=\mathrm{B}(m, n) \mathbf{p}_{m, n}+\mathbf{z}_{m, n} .
$$

For a given Signal-to-Clutter Ratio (SCR), denoted by $\mathrm{SCR}_{m, n}$ the amplitude of $\mathrm{B}(m, n)$ is given by:

$$
|\mathrm{B}(m, n)|=\sqrt{\mathrm{SCR}_{m, n} \cdot \sigma^{2}} .
$$




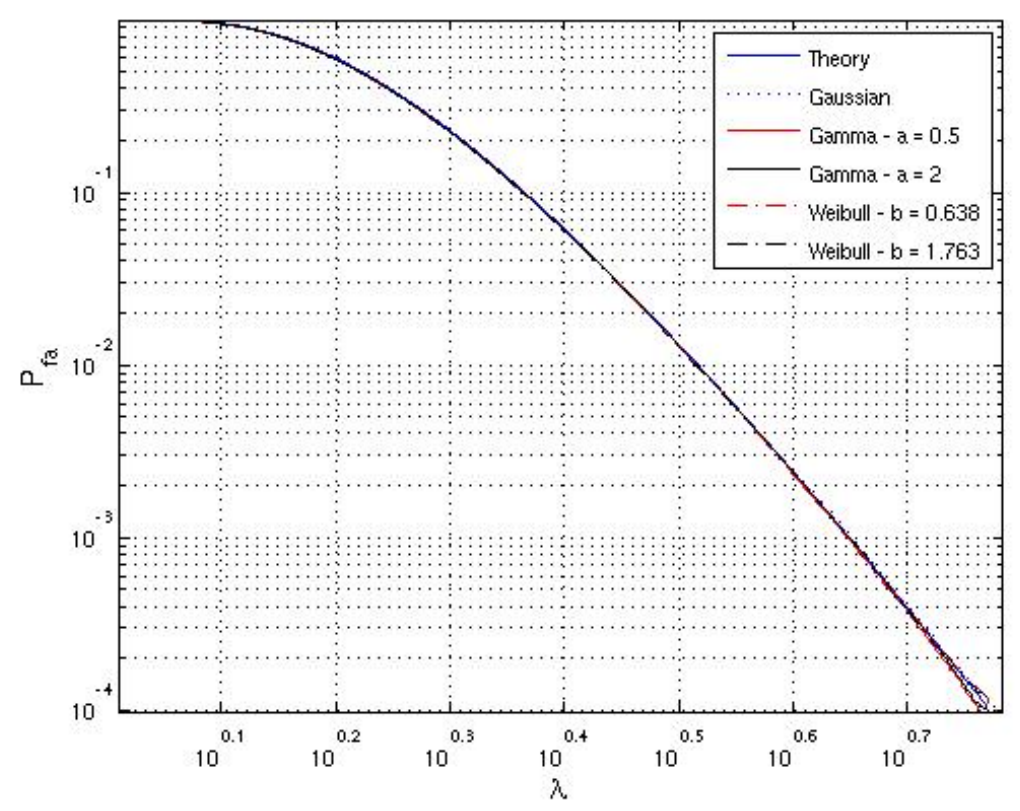

Fig. 2. $P_{f a}$ against detection threshold $\lambda$ for theoretical calculations and Monte-Carlo simulations under Gaussian clutter and Gamma and Weibull texture.

If $|\mathrm{B}(m, n)|$ is considered to be different for each subarray, a multi-dimensional graph would be necessary to represent the detection performance and it would be very difficult to interpret the results. Hence, in this paper, we consider that $|\mathrm{B}(m, n)|$ is the same for all $m$ and $n$.

For comparison, the OGD detector which is optimum under Gaussian clutter is considered. According to [7] and [25], the OGD detector extended to MIMO case is given by:

$$
\Lambda(\mathbf{Y})=\sum_{i=1}^{K} \frac{\left|\mathbf{p}_{i}^{\dagger} \mathbf{M}_{i}^{-1} \mathbf{y}_{i}\right|^{2}}{\mathbf{p}_{i}^{\dagger} \mathbf{M}_{i}^{-1} \mathbf{p}_{i}},
$$

where $\mathbf{Y}=\left[\mathbf{y}_{1}, \cdots, \mathbf{y}_{K}\right]$ and $\mathbf{M}_{i}$ which is the covariance matrix of $\mathbf{y}_{i}$, is assumed to be known. Under Gaussian clutter, this detector has a Chi-square distribution with $2 K$ degrees of freedom, denoted by $\chi_{2 K}^{2}$.

The same parameters as before are used (see Table I and II). The $P_{f a}$ is set to be 0.001 and $N_{r}=10^{4}$. The detection performance for MIMO GLRT-LQ and MIMO OGD under K-distributed clutter is shown in Fig. 3. The SCR values in the $y$-axis are for only one single subarray. The clutter is more impulsive for smaller values of $a$. We see that when $a=0.5$, MIMO GLRT-LQ performs much better than MIMO OGD. This is because for MIMO OGD, the big variation of clutter power results in a high detection threshold to maintain the same $P_{f a}$. The increase in threshold results in a drop in detection performance. 
On the other hand, there is a normalizing term $\mathbf{y}^{\dagger} \mathbf{M}^{-1} \mathbf{y}$ in MIMO GLRT-LQ to take into account this variation. When the clutter is less impulsive, it becomes more similar to Gaussian clutter and the performance of both detectors tends to be similar.

The performance results for clutter with Weibull-distributed texture can be seen in Fig. 4. For this case, the clutter is more impulsive for smaller values of $b$. As expected, MIMO GLRT-LQ performs much better than MIMO OGD when the clutter is impulsive and almost the same when the clutter is less impulsive.

In Fig. 5, we see that the new detector works slightly worse than MIMO OGD under Gaussian clutter. This is expected since MIMO OGD is the optimal detector under Gaussian clutter. However, MIMO GLRT-LQ is much more robust as it maintains good detection performance for different types of clutter.

\section{Adaptive Mimo non-Gaussian Detector}

\section{A. Derivation}

In the previous sections, we assumed that the covariance matrix was known. However, it is usually unknown in reality. Hence we consider the adaptive version of the detector; i.e. the covariance matrix $\mathbf{M}_{m, n}$ is replaced by its estimate $\hat{\mathbf{M}}_{m, n}$ :

$$
\hat{\Lambda}(\mathbf{Y})=\prod_{m, n}\left[\frac{1}{1-\frac{\left|\mathbf{p}_{m, n}^{\dagger} \hat{\mathbf{M}}_{m, n}^{-1} \mathbf{y}_{m, n}\right|^{2}}{\left(\mathbf{p}_{m, n}^{\dagger} \hat{\mathbf{M}}_{m, n}^{-1} \mathbf{p}_{m, n}\right)\left(\mathbf{y}_{m, n}^{\dagger} \hat{\mathbf{M}}_{m, n}^{-1} \mathbf{y}_{m, n}\right)}}\right]^{M_{m} N_{n}} \underset{\mathrm{H}_{0}}{\stackrel{\mathrm{H}_{1}}{\gtrless}} \eta .
$$

Under Gaussian clutter, the classical SCM is the ML estimate, given by:

$$
\hat{\mathbf{M}}_{m, n}=\frac{1}{L_{r}} \sum_{l=1}^{L_{r}} \mathbf{x}_{m, n}(l) \mathbf{x}_{m, n}^{\dagger}(l),
$$

where $\mathbf{x}_{m, n}(l) \sim \mathcal{C N}\left(\mathbf{0}, \mathbf{M}_{m, n}\right)$ are the secondary data which are independent and identically distributed. $L_{r}$ is the number of secondary data used to estimate the SCM. SCM follows the complex Wishart distribution, denoted as $\mathcal{C W}\left(L_{r}, L ; \mathbf{M}_{m, n}\right)$.

However, under non-Gaussian clutter, $\mathbf{y}_{m, n}(l)=\sqrt{\tau_{m, n}} \mathbf{x}_{m, n}(l)$ :

$$
\begin{aligned}
\hat{\mathbf{M}}_{m, n} & =\frac{1}{L_{r}} \sum_{l=1}^{L_{r}} \mathbf{y}_{m, n}(l) \mathbf{y}_{m, n}^{\dagger}(l), \\
& =\frac{1}{L_{r}} \sum_{l=1}^{L_{r}} \tau_{m, n} \mathbf{x}_{m, n}(l) \mathbf{x}_{m, n}^{\dagger}(l),
\end{aligned}
$$

we see that the SCM is no longer the ML estimate. Instead, we consider the FPE [21], [22], [23]. In [23], it has been shown that the FPE is unique up to a scalar factor. Here, due to the matrix normalization, 


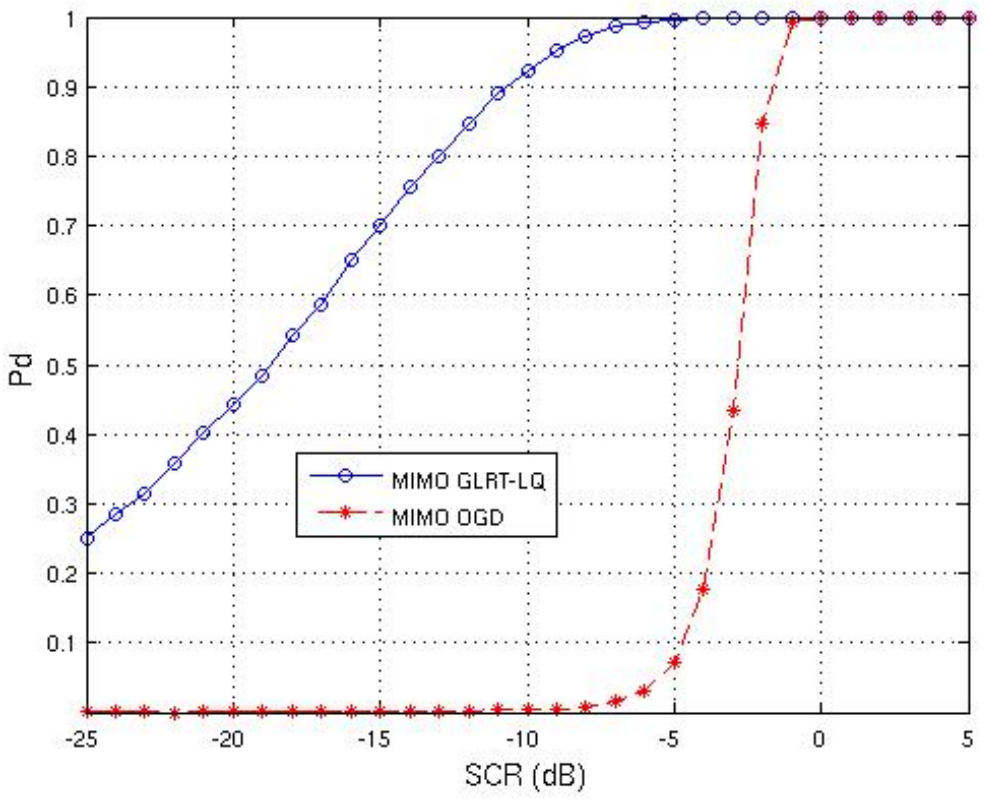

(a) $a=0.5$

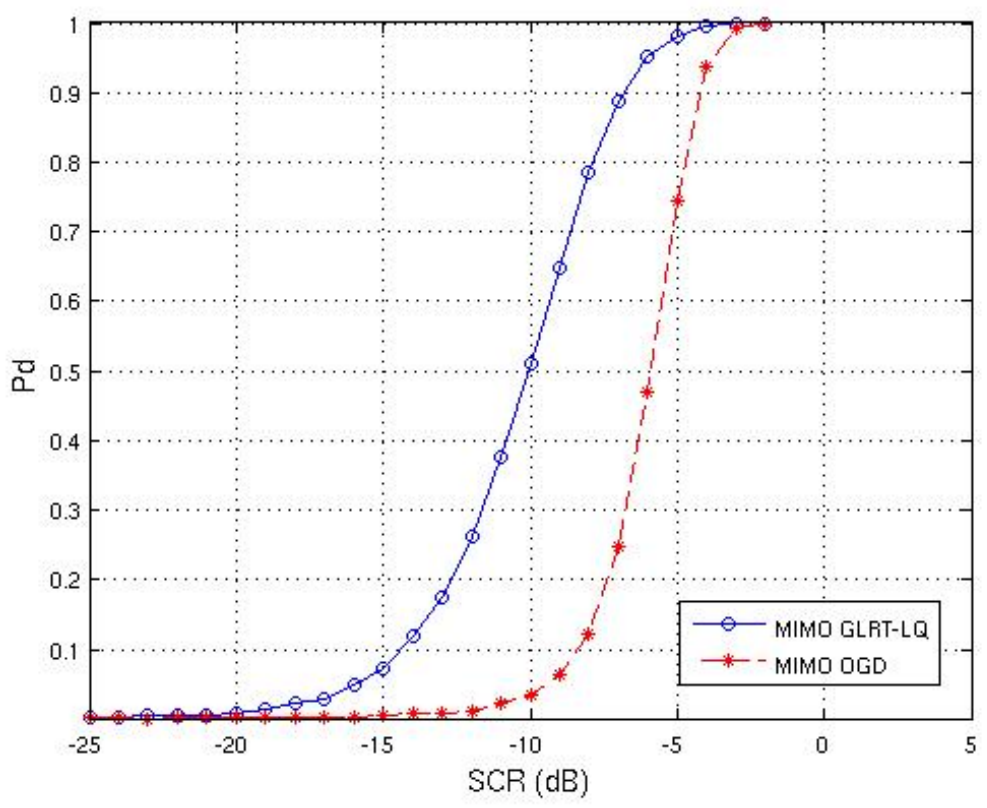

(b) $a=2$

Fig. 3. $P_{d}$ against SCR for Monte-Carlo simulations where the texture has a Gamma distribution with different parameters, with $P_{f a}=0.001$. 


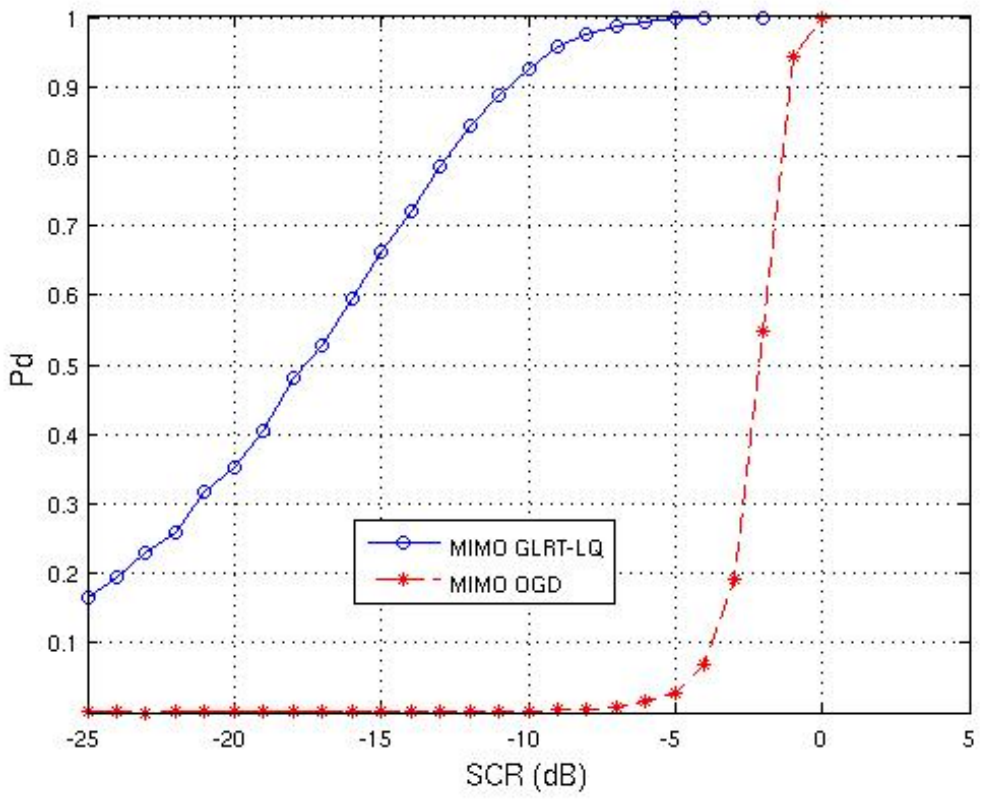

(a) $b=0.658$

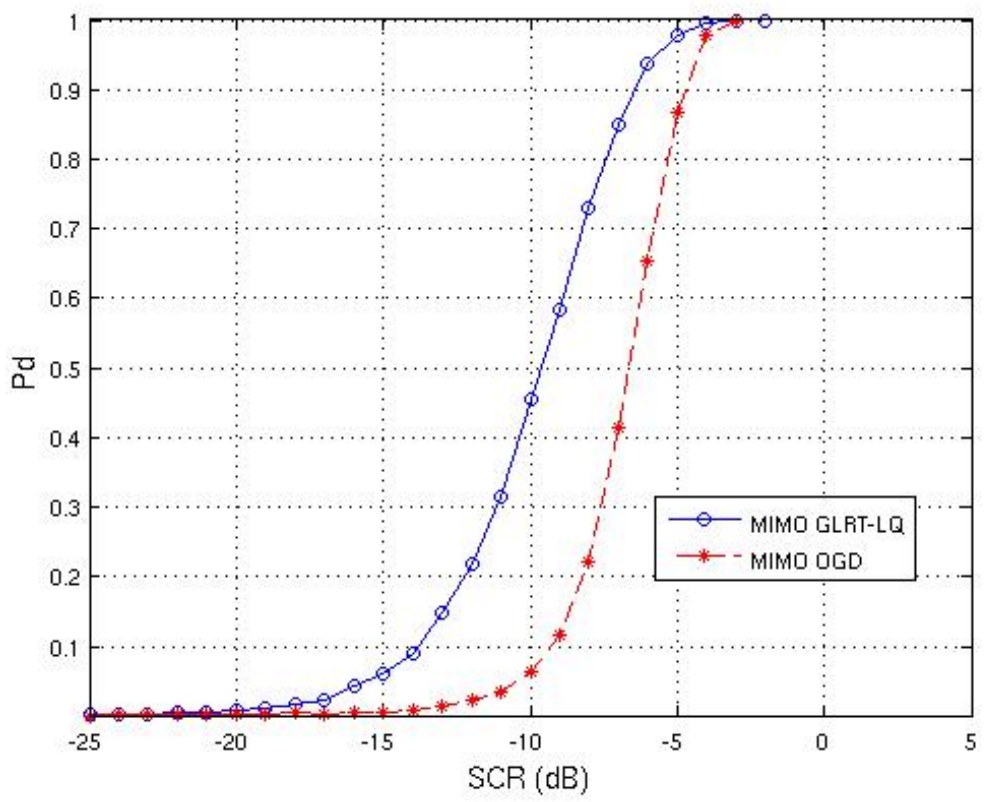

(b) $a=1.763$

Fig. 4. $P_{d}$ against SCR for Monte-Carlo simulations where the texture has a Weibull distribution with different parameters, with $P_{f a}=0.001$. 


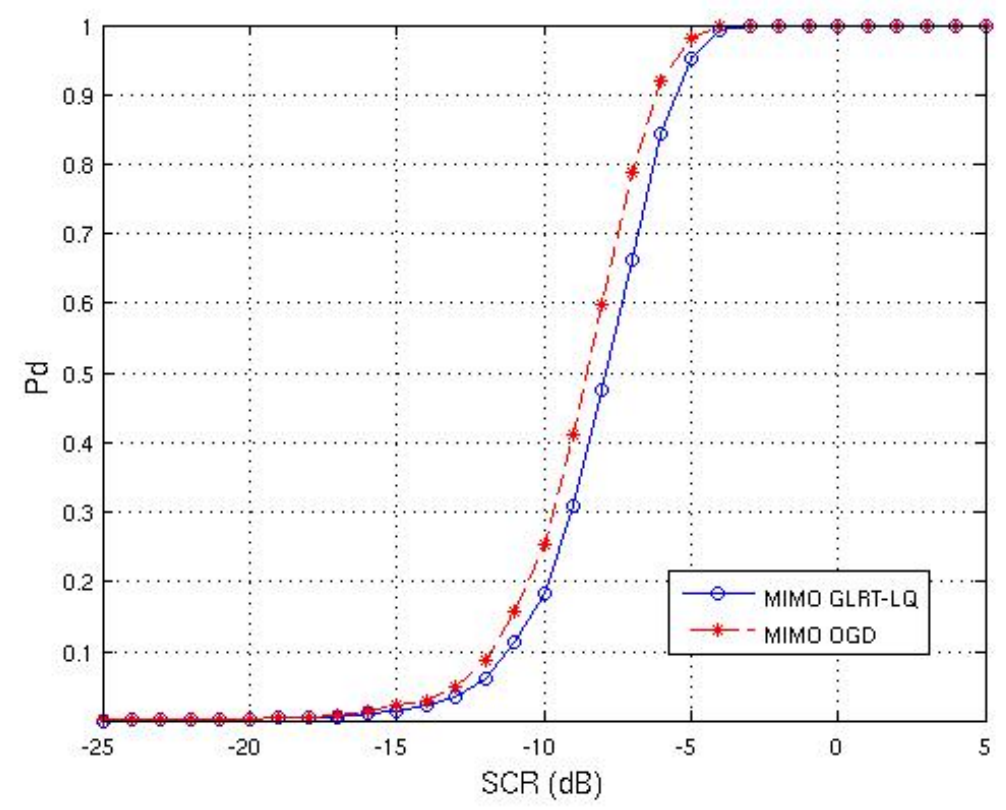

Fig. 5. $P_{d}$ against SNR for Monte-Carlo simulations where the clutter is Gaussian, with $P_{f a}=0.001$.

the resulting FPE is unique and it is defined as the unique solution of the equation:

$$
\hat{\mathbf{M}}_{m, n}=\frac{L}{L_{r}} \sum_{l=1}^{L_{r}} \frac{\mathbf{y}_{m, n}(l) \mathbf{y}_{m, n}^{\dagger}(l)}{\mathbf{y}_{m, n}^{\dagger}(l) \hat{\mathbf{M}}_{m, n}^{-1} \mathbf{y}_{m, n}(l)} .
$$

Due to the normalizing term in the denominator, the FPE does not depend on the texture of the clutter. Moreover, the FPE is the ML estimate when the texture is deterministic but unknown [23]. In the case where the texture is a random variable, the FPE is the approximate ML estimate [21], [22].

The FPE is computed using the following iterative algorithm:

$$
\hat{\mathbf{M}}_{m, n}(k+1)=\frac{L}{L_{r}} \sum_{l=1}^{L_{r}} \frac{\mathbf{y}_{m, n}(l) \mathbf{y}_{m, n}^{\dagger}(l)}{\mathbf{y}_{m, n}^{\dagger}(l) \hat{\mathbf{M}}_{m, n}^{-1}(k) \mathbf{y}_{m, n}(l)} .
$$

The solution converges towards the FPE irregardless of the choice of the initial matrix $\hat{\mathbf{M}}_{m, n}(0)$ as shown in [23]. One obvious choice is $\mathbf{I}$ which will give the normalized SCM as the first estimate. Consequently, the detector is also matrix-CFAR when the FPE is used [32].

More importantly, it has been shown in [24] that the asymptotic distribution of $\hat{\mathbf{M}}_{F P}$ is the same as that of the SCM with $\frac{L}{L+1} L_{r}$ secondary data under Gaussian clutter. 
d) $\tilde{M}=1$ and $\tilde{N}=1$ (Adaptive

As before, we begin with the case where $\tilde{M}=1$ and $\tilde{N}=1$. The dimension of the received signal is $L=M_{1} N_{1}$. As there is only one received signal and steering vector, let them be denoted simply by $\mathbf{y}=\mathbf{y}_{1,1}$ and $\mathbf{p}=\mathbf{p}_{1,1}$. The adaptive detector is given by:

$$
\hat{\Lambda}_{1}(\mathbf{y})=\left[\frac{1}{1-\frac{\left|\mathbf{p}^{\dagger} \hat{\mathbf{M}}^{-1} \mathbf{y}\right|^{2}}{\left(\mathbf{p}^{\dagger} \hat{\mathbf{M}}^{-1} \mathbf{p}\right)\left(\mathbf{y}^{\dagger} \hat{\mathbf{M}}^{-1} \mathbf{y}\right)}}\right] \quad \gtrless_{\mathrm{H}_{0}}^{\mathrm{H}_{1}} \quad \sqrt[L]{\eta}=\lambda .
$$

According to [26], under $\mathrm{H}_{0}$, considering Gaussian clutter and using the SCM as the estimated covariance matrix, $\hat{\Lambda}_{1}(\mathbf{y})$ can be expressed with a random variable $\hat{F}$ which in turn depends on another random variable $B$ :

$$
\hat{\Lambda}_{1}(\mathbf{y})=1+\hat{F} \text {. }
$$

The distributions of the two variables are as follows: $\hat{F} \mid B \sim \beta_{1, L_{r}-L+1}^{1}$ and $B \sim \beta_{L_{r}-L+2, L-1}^{1}$. The PDF of $\beta_{a, b}^{1}$ and $\beta_{a, b}^{2}$ are defined in [27] as:

$$
\begin{array}{r}
\beta_{a, b}^{1}(x)=\frac{\Gamma(a+b)}{\Gamma(a) \Gamma(b)} x^{a-1}(1-x)^{b-1} \mathbb{1}_{[0,1]}(x), \\
\beta_{a, b}^{2}(x)=\frac{\Gamma(a+b)}{\Gamma(a) \Gamma(b)} \frac{x^{a-1}}{(1+x)^{a+b}}, \quad x>0 .
\end{array}
$$

Here, we consider instead non-Gaussian clutter modeled as a SIRV. Using the FPE, $\hat{\Lambda_{1}}(\mathbf{y})$ can still be expressed with a conditional random variable $\hat{F} \mid B$ but with $L_{r}$ replaced by $\frac{L}{L+1} L_{r}$. Hence the distribution becomes $\hat{F} \mid B \sim \beta_{1, a-1}^{1}$ with $B \sim \beta_{a, b-a-1}^{1}$ where $a=\frac{L}{L+1} L_{r}-L+2$ and $b=\frac{L}{L+1} L_{r}+2$.

Using Eqn. (18) and (19), we obtain the PDF of $\hat{F}$ :

$$
\begin{aligned}
f(\hat{F}) & =\int_{0}^{1} \frac{\Gamma(a)}{\Gamma(a-1)} \frac{1-B}{(1+\hat{F}(1-B))^{a}} \frac{\Gamma(b-1)}{\Gamma(a) \Gamma(b-a-1)} B^{a-1}(1-B)^{b-a-2} d B, \\
& =\frac{\Gamma(b-1)}{\Gamma(a-1) \Gamma(b-a-1)} \frac{1}{(1+\hat{F})^{a}} \int_{0}^{1} \frac{B^{a-1}(1-B)^{b-a-1}}{\left(1-\frac{\hat{F}}{1+\hat{F}} B\right)^{a}} d B, \\
& =\frac{\Gamma(b-1)}{\Gamma(a-1) \Gamma(b-a-1)} \frac{\Gamma(a) \Gamma(b-a)}{\Gamma(b)} \frac{1}{(1+\hat{F})^{a}}{ }_{2} \mathrm{~F}_{1}\left(a, a, b, \frac{\hat{F}}{1+\hat{F}}\right), \\
& =\frac{(a-1)(b-a-1)}{(b-1)} \frac{1}{(1+\hat{F})^{a}}{ }_{2} \mathrm{~F}_{1}\left(a, a, b, \frac{\hat{F}}{1+\hat{F}}\right),
\end{aligned}
$$

where ${ }_{2} \mathrm{~F}_{1}(a, b, c, x)$ is the hypergeometric function as defined in [27]:

$$
{ }_{2} \mathrm{~F}_{1}(a, b, c, x)=\frac{\Gamma(c)}{\Gamma(b) \Gamma(c-b)} \int_{0}^{1} \frac{t^{b-1}(1-t)^{c-b-1}}{(1-t x)^{a}} d t .
$$


Letting $\hat{\Lambda}_{1}(\mathbf{y})=u_{1}=1+\hat{F}$, the PDF becomes:

$$
f\left(u_{1}\right)=\frac{(a-1)(b-a-1)}{(b-1)} u_{1}^{-a}{ }_{2} \mathrm{~F}_{1}\left(a, a, b, 1-\frac{1}{u_{1}}\right) .
$$

To obtain the $P_{f a}$, we integrate the PDF from $\lambda$ to $\infty$ :

$$
P_{f a}=\int_{\lambda}^{\infty} f\left(u_{1}\right) d u_{1}
$$

Through a change of variable $x=1-\frac{1}{u_{1}}$ and the Euler Transformation for hypergeometric functions (Eqn. (15.3.4) in [27]), we get:

$$
\begin{aligned}
P_{f a} & =\int_{1-1 / \lambda}^{1} \frac{(a-1)(b-a-1)}{(b-1)}(1-x)^{a}{ }_{2} \mathrm{~F}_{1}(a, a, b, x) \frac{1}{(1-x)^{2}} d x, \\
& =\int_{1-1 / \lambda}^{1} \frac{(a-1)(b-a-1)}{(b-1)}{ }_{2} \mathrm{~F}_{1}\left(a, b-a, b, \frac{x}{x-1}\right) \frac{1}{(1-x)^{2}} d x .
\end{aligned}
$$

Consider the hypergeometric function $F={ }_{2} \mathrm{~F}_{1}\left(a-1, b-a-1, b-1, \frac{x}{x-1}\right)$, the derivative of $F$ is then:

$$
\frac{d F}{d x}=\frac{(a-1)(b-a-1)}{(b-1)}{ }_{2} \mathrm{~F}_{1}\left(a, b-a, b, \frac{x}{x-1}\right) \frac{-1}{(1-x)^{2}} .
$$

Using this derivative, we can obtain the final expression for $P_{f a}$ :

$$
\begin{aligned}
P_{f a} & =\int_{1-1 / \lambda}^{1}-\frac{d F}{d x} d x \\
& ={ }_{2} \mathrm{~F}_{1}(a-1, b-a-1, b-1,1-\lambda) .
\end{aligned}
$$

From Eqns. (21) and (22), we see that the distribution of the detector and the $P_{f a}$, respectively, depends only on $L_{r}$ and $L$. This means that the adaptive detector is also CFAR.

As $L_{r} \rightarrow \infty, a-1 \approx b-1$. Using the identity Eqn. (15.1.8) in [27], ${ }_{2} \mathrm{~F}_{1}(a, b, b, z)={ }_{2} \mathrm{~F}_{1}(b, a, b, z)=$ $(1-z)^{-a}$, we see that, asymptotically, $P_{f a}$ tends to

$$
P_{f a} \rightarrow \lambda^{-(b-a-1)}=\lambda^{-L+1},
$$

which is the same expression as that for the case where the covariance matrix is known and $K=1$.

e) $\tilde{M} \geq 1, \tilde{N} \geq 1$ and $M_{m} N_{n}=L$ (Adaptive)

As before, we consider the product of all the individual detection tests for each subsystem $u=\prod_{i=1}^{K} u_{i}$. The joint density function is given by:

$$
f\left(u_{1}, \ldots, u_{K}\right)=\left(\frac{(a-1)(b-a-1)}{(b-1)}\right)^{K} \prod_{i=1}^{K} u_{i}^{-a}{ }_{2} \mathrm{~F}_{1}\left(a, a, b, 1-\frac{1}{u_{i}}\right) .
$$


As before, we consider the bijective function from $\left(u_{1}, \ldots, u_{K}\right)$ to $\left(u, v_{1}, \ldots, v_{K-1}\right)$ :

$$
\begin{aligned}
& f\left(u, v_{1}, \ldots, v_{K-1}\right)= \\
& \quad\left(\frac{(a-1)(b-a-1)}{(b-1)}\right)^{K} u^{-a} \prod_{i=1}^{K-1}{ }_{2} \mathrm{~F}_{1}\left(a, a, b, 1-\frac{1}{u_{i}}\right){ }_{2} \mathrm{~F}_{1}\left(a, a, b, 1-\frac{\prod_{j=1}^{K-1} u_{j}}{u}\right) \frac{1}{\prod_{i=1}^{K-1} v_{i}} .
\end{aligned}
$$

Due to the complexity of the above expression, the PDF of $f(u)$ and consequently $P_{f a}$ has not been obtained analytically. Instead, $P_{f a}$ is computed empirically using Monte Carlo simulations.

\section{B. Simulation Results (Adaptive)}

The same parameters as before (Table I and II) are used. To study the effects of the estimation of the covariance matrix on the detection performance, we consider 2 cases: $L_{r}=2 L$ and $L_{r}=20 L$ to compute $\hat{\mathbf{M}}_{i}$ using the Fixed Point algorithm, and compare them to the case where $\mathbf{M}_{i}$ is known.

As a rule of thumb, it is considered that $L_{r}$ has to be at least $2 L$ and this will give a loss of approximately $3 \mathrm{~dB}$ in detection performance [33]. On the other hand, $L_{r}=20 L$ has been chosen to approach the asymptotic case where $\mathbf{M}$ is known.

The FPE is obtained using Eqn. (17) with 10 iterations. However, from an operational point of view, it suffices to compute the estimate with 4 or 5 iterations to achieve a relative error of $10^{-2}$ [23].

As expected, when $L_{r}$ is large, the detection performance of the adaptive detector tends towards that of the detector where the covariance matrices are known (Fig. 6).

\section{f) Under Gaussian Clutter (Adaptive)}

For comparison, the MIMO Adaptive Matched Filter (MIMO AMF), the adaptive version of MIMO-OGD, has been considered. It is obtained simply by replacing the estimated covariance matrix in Eqn. (13):

$$
\hat{\Lambda}(\mathbf{Y})=\sum_{i=1}^{K} \frac{\left|\mathbf{p}_{i}^{\dagger} \hat{\mathbf{M}}_{i}^{-1} \mathbf{y}_{i}\right|^{2}}{\mathbf{p}_{i}^{\dagger} \hat{\mathbf{M}}_{i}^{-1} \mathbf{p}_{i}} .
$$

The SCM given in Eqn. (15) is used in the MIMO AMF since it is the ML estimate under Gaussian clutter. In Fig. 7, we have the detection performance, under Gaussian clutter, of both the adaptive MIMO GLRT-LQ using FPE and the MIMO AMF using SCM. Even in this case, the estimation of the covariance matrix does not affect the performance of the adaptive MIMO GLRT-LQ much and it remains comparable to that of the MIMO AMF.

According to [34], under Gaussian clutter, the AMF is expected to perform worse than Kelly's Test [35] as the signal vector $\mathbf{y}_{i}$ is not used in the estimation of the covariance matrix. The Kelly's Test, for the 


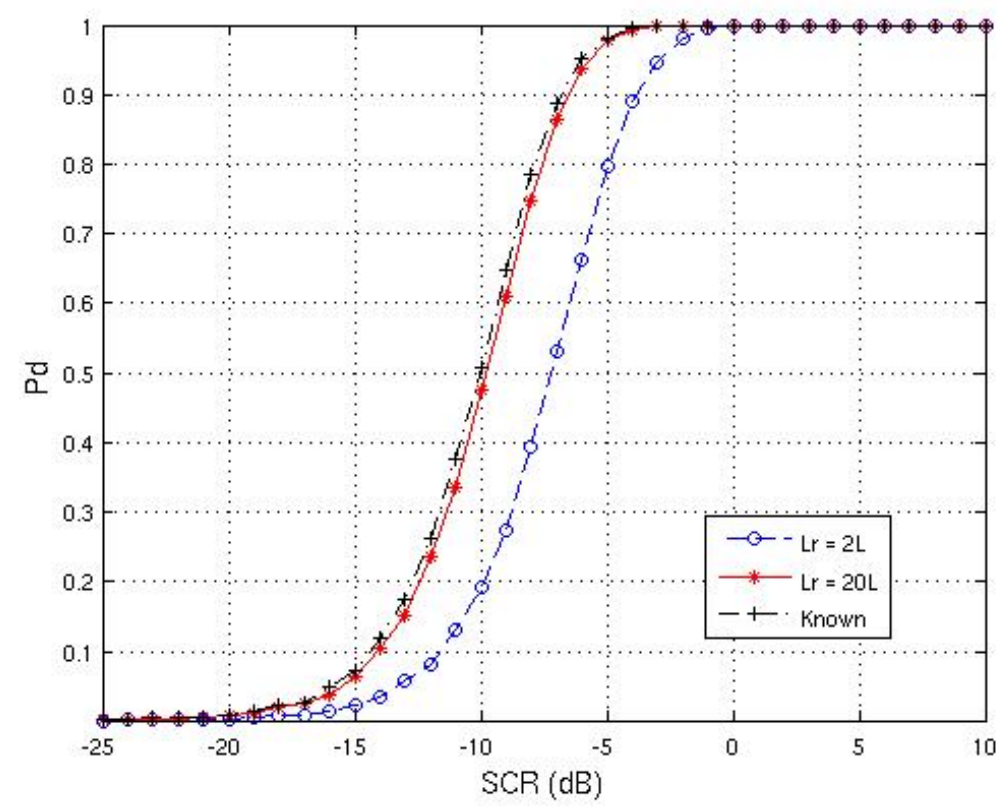

Fig. 6. $\quad P_{d}$ against SCR for Monte-Carlo simulations with the adaptive MIMO GLRT-LQ using FPEs and clutter with Gamma-distributed texture $(a=2)$.

case where $K=1$, is given by:

$$
\hat{\Lambda}\left(\mathbf{y}_{1}\right)=\frac{\left|\mathbf{p}_{1}^{\dagger} \hat{\mathbf{M}}_{1}^{-1} \mathbf{y}_{1}\right|^{2}}{\left(\mathbf{p}_{1}^{\dagger} \hat{\mathbf{M}}_{1}^{-1} \mathbf{p}_{1}\right)\left(1+\frac{1}{L_{r}} \mathbf{y}_{1}^{\dagger} \hat{\mathbf{M}}_{1}^{-1} \mathbf{y}_{1}\right)} .
$$

When $L_{r}$ is large, Kelly's Test is similar to the AMF. However, when $L_{r}$ is small, the term $\mathbf{y}_{1}^{\dagger} \hat{\mathbf{M}}_{1}^{-1} \mathbf{y}_{1}$ is no longer negligible and Kelly's Test is more similar to the adaptive GLRT-LQ test. Thus, in Fig. 7, we see that the performance of the adaptive MIMO GLRT-LQ is actually slightly better than that of the MIMO AMF when $L_{r}=2 L$.

\section{g) Under Non-Gaussian Clutter (Adaptive)}

Fig. 8 and Fig. 9 show the detection performance for both the adaptive MIMO GLRT-LQ with FPE and the MIMO AMF with SCM when the texture has Gamma and Weibull distribution, respectively. As expected, the adaptive MIMO GLRT-LQ performs much better than the MIMO AMF when the clutter is impulsive. When the clutter is less impulsive, the detection performance of both detectors is similar but the adaptive MIMO GLRT-LQ still works better. Moreover, the MIMO AMF is more sensitive to the estimation of the covariance matrix than the adaptive MIMO GLRT-LQ. This is not surprising since the 


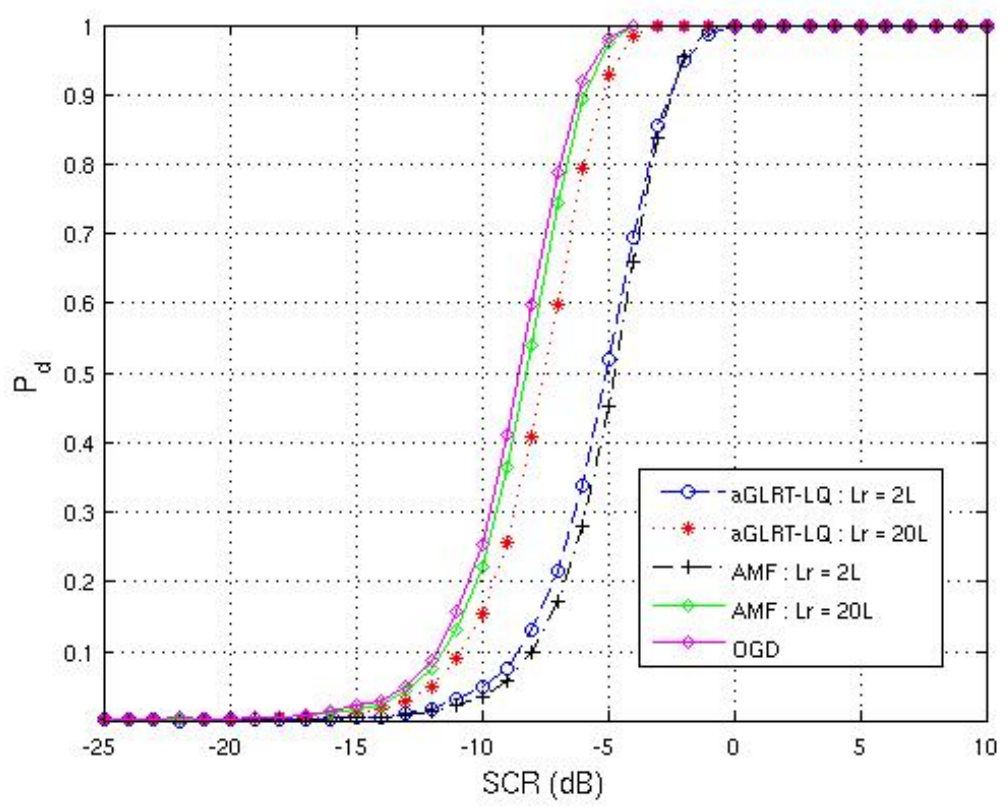

Fig. 7. $P_{d}$ against SCR for Monte-Carlo simulations for both the adaptive MIMO GLRT-LQ using FPEs and the MIMO AMF using SCM with Gaussian clutter. $P_{d}$ against SCR for MIMO OGD is given as reference. $P_{f a}=0.001$.

SCM is no longer the ML estimate under non-Gaussian clutter.

\section{Conclusions}

The CFAR GLRT-LQ detector for detection under non-Gaussian clutter has been extended to the MIMO case where all subarrays are considered jointly as a system such that only one detection threshold is used. Theoretical performance for the new detector is also derived and validated using Monte Carlo simulations. Detection performance is then analyzed through simulations. Compared to the classical OGD detector, the new detector shows significant improvements in detection performance under non-Gaussian clutter especially in very impulsive clutter. It has a slight loss in performance when the clutter is Gaussian. This is expected since the MIMO OGD is the optimal detector under Gaussian clutter. However, the robustness of the MIMO GLRT-LQ detector under non-Gaussian clutter more than compensates this slight loss under Gaussian clutter.

Next, the adaptive version of this new detector is considered. The FPE is used to estimate the covariance matrix as the classical SCM no longer works under non-Gaussian clutter. The theoretical performance of the adaptive version is shown to be texture-CFAR and matrix-CFAR for the case where there is only one subarray. On top of that, the detection performance tends to that of the case where the covariance 


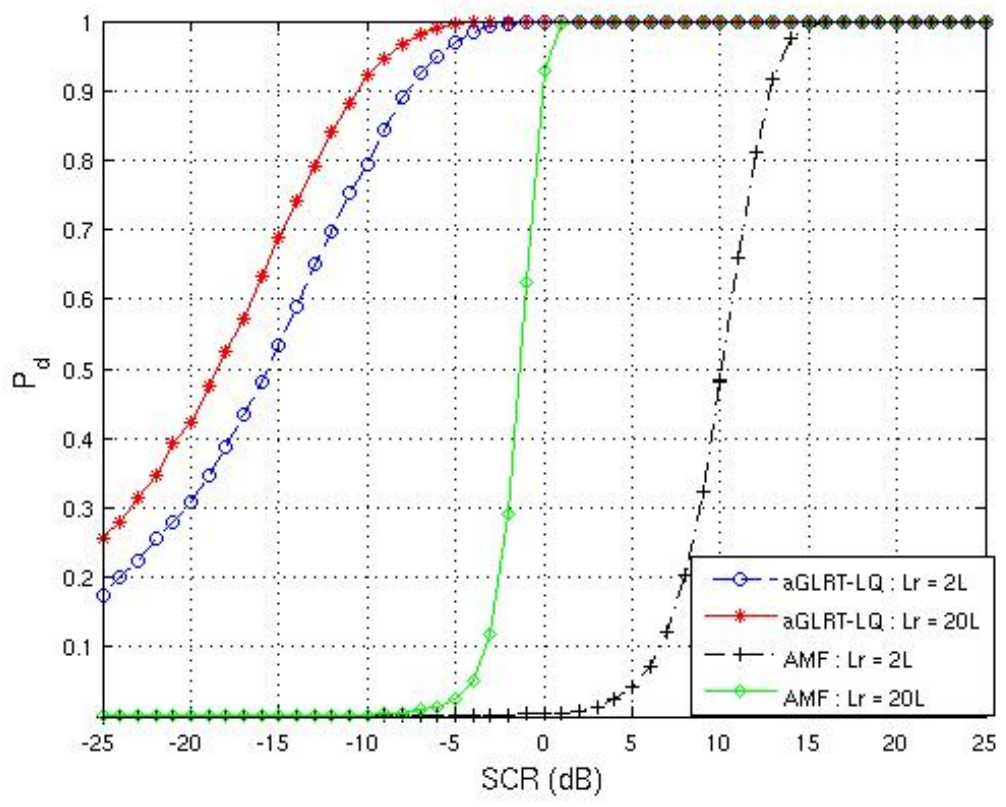

(a) $a=0.5$

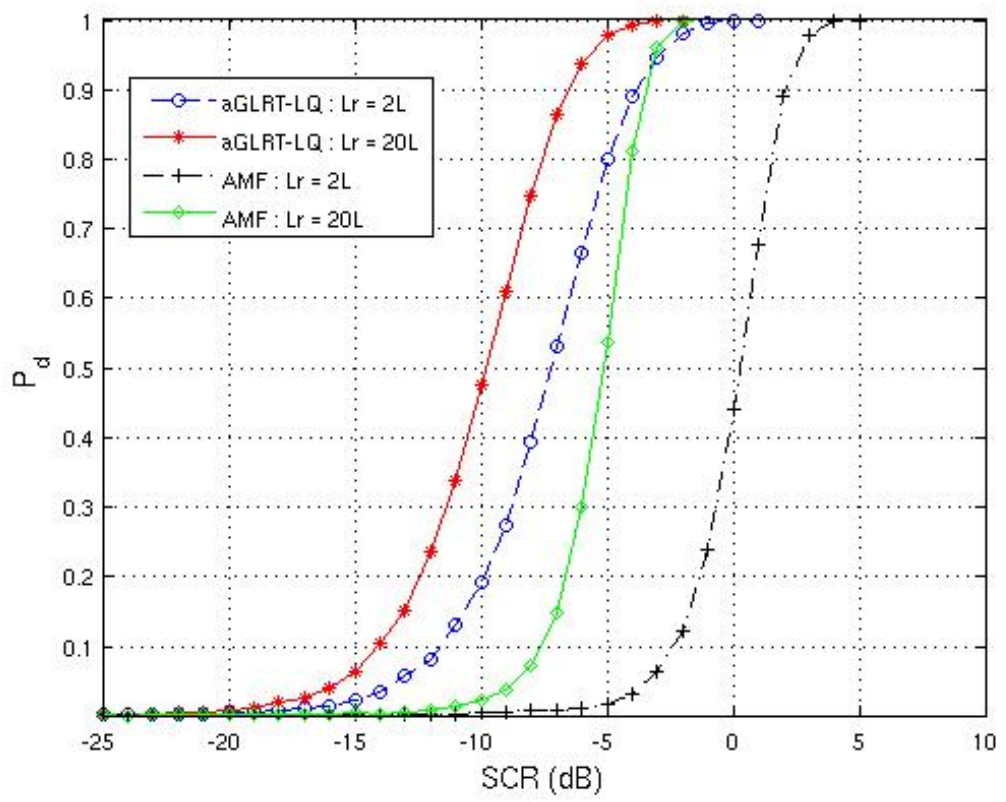

(b) $a=2$

Fig. 8. $\quad P_{d}$ against SCR for Monte-Carlo simulations for both the adaptive MIMO GLRT-LQ using FPEs and the MIMO AMF using SCM with K-distributed clutter. $P_{f a}=0.001$. 


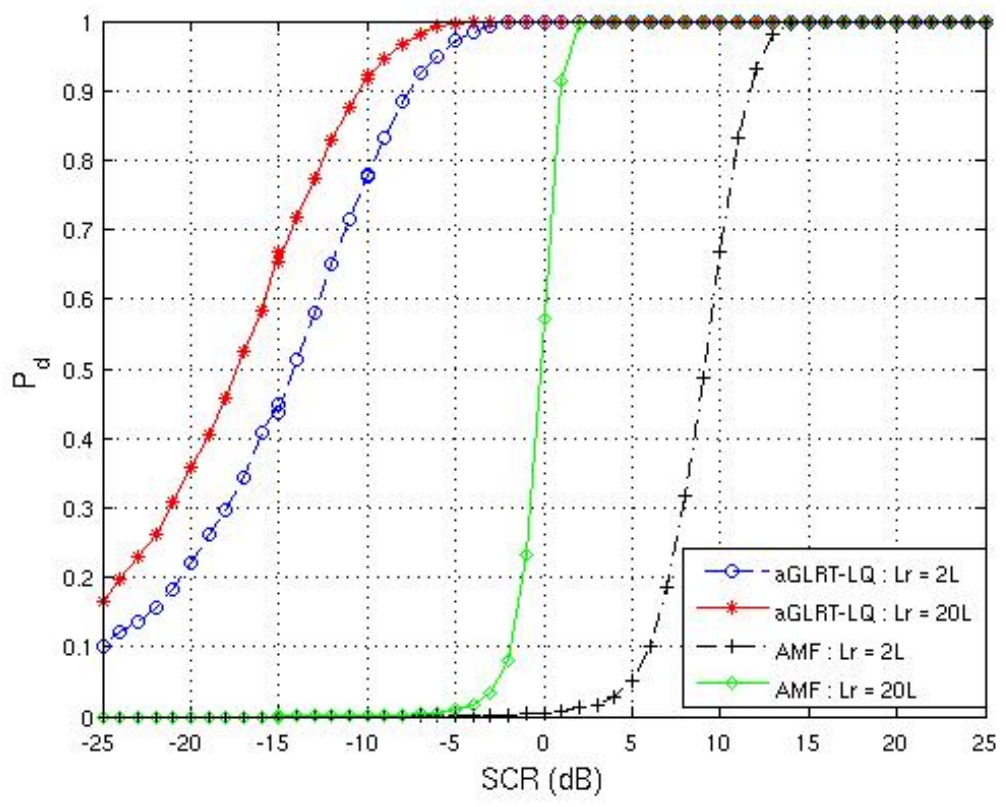

(a) $b=0.658$

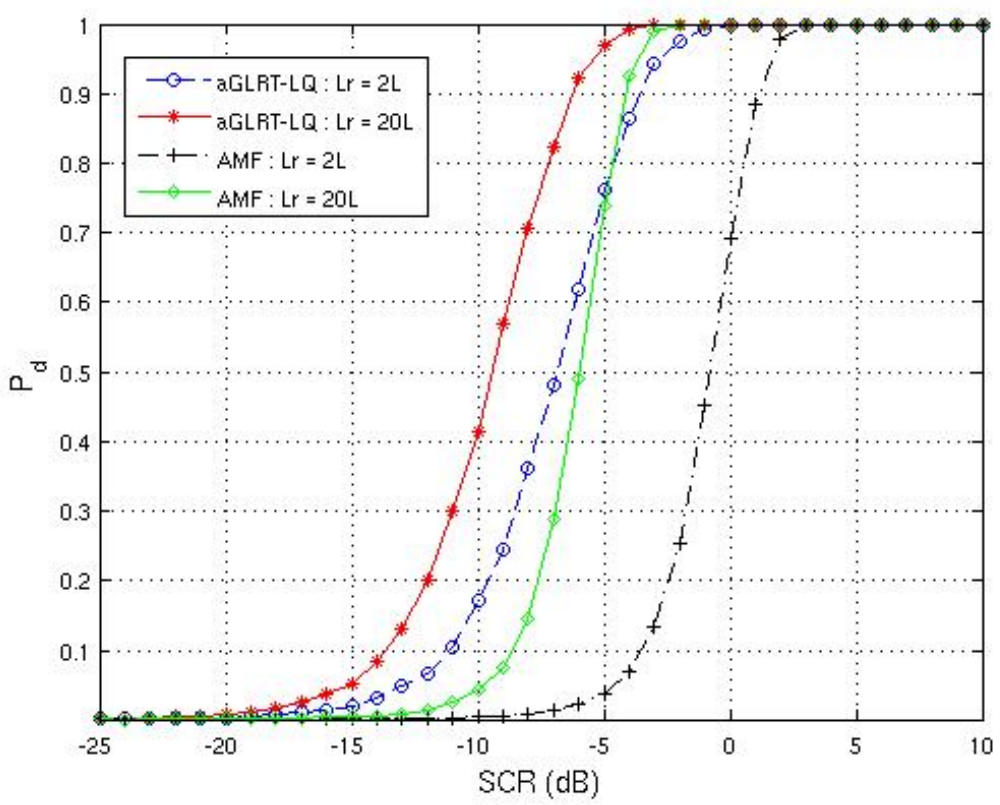

(b) $b=1.763$

Fig. 9. $P_{d}$ against SCR for Monte-Carlo simulations for both the adaptive MIMO GLRT-LQ using FPEs and the MIMO AMF using SCM and clutter with Weibull-distributed texture. $P_{f a}=0.001$. 
matrix is known when the number of secondary data is large. Due to the complexity of equations, the case where there are more than one subarray is studied only empirically using simulations.

The detection performance of the adaptive non-Gaussian detector using the FPE is then compared to the MIMO AMF using the SCM through simulations. As expected, the new detector performs much better under non-Gaussian clutter, especially when the clutter is very impulsive and it has comparable performance under Gaussian clutter.

The main conclusion is that it is always preferable to use the adaptive GLRT-LQ with the FPE, whatever the clutter distribution, because of the robustness of these tools with respect to the covariance matrix and the texture. Even in the case where the clutter is Gaussian for all subarrays, the covariance matrix and clutter power for each subarray is expected to be different. Hence it is still better to use the new non-Gaussian detector.

\section{REFERENCES}

[1] J. Li and P. Stoica, MIMO Radar Signal Processing, Wiley, 1st edition, 2009.

[2] E. Fishler, A. Haimovich, R. Blum, L. Cimini, D. Chizhik, and R. Valenzuela, "Performance of MIMO radar systems: Advantages of angular diversity," in Proc. 38th Asilomar Conference on Signals, Systems and Computers, Pacific Grove, USA, Nov 2004, pp. 305-309.

[3] N. H. Lehmann, E. Fishler, A. Haimovich, R. Blum, D. Chizhik, and L. Cimini, "Evaluation of transmit diversity in MIMO-radar direction finding,” IEEE Trans. Sig. Proc., vol. 55, no. 5, pp. 2215-2225, May 2007.

[4] N. H. Lehmann, A. Haimovich, R. Blum, and L. Cimini, "High resolution capabilities of MIMO radar," in Proc. 40th Asilomar Conference on Signals, Systems and Computers, Pacific Grove, USA, Oct 2006.

[5] C-Y. Chen and P. P. Vaidyanathan, "A subspace method for MIMO radar space-time adaptive processing," in Proc. ICASSP, April 2007.

[6] J. Li and P. Stoica, "MIMO radar with colocated antennas," IEEE Sig. Proc. Magazine, vol. 24, no. 5, pp. 106-114, Sept 2007.

[7] E. Fishler, A. Haimovich, R. Blum, and L. J. Cimini, "Spatial diversity in radars - models and detection performance," IEEE Trans. Sig. Proc., vol. 54, no. 54, pp. 823-838, March 2006.

[8] A. M. Haimovich, R. S. Blum, and L. J. Cimini Jr, "MIMO radar with widely separated antennas," IEEE Sig. Proc. Magazine, vol. 25, no. 1, pp. 116-129, Jan 2008.

[9] L. Xu, J. Li, and P. Stoica, "Radar imaging via adaptive MIMO techniques," in Proc. 14th European Signal Processing Conference, Florence, Italy, Sept 2006.

[10] J. Li, "Multi-Input Multi-Output (MIMO) radar - diversity means superiority," Annual report, Office of Naval Research, Oct 2007.

[11] J. B. Billingsley, “Ground clutter measurements for surface-sited radar," Technical Report 780, MIT, Feb 1993.

[12] S. Watts, "Radar detection prediction in sea clutter using the compound K-distribution model," IEE Proc. F, vol. 132, no. 7, pp. 613-620, 1985.

[13] T. Nohara and S. Haykin, "Canada east coast trials and the K-distribution," IEE Proc. F, vol. 138, no. 2, pp. 82-88, 1991. 
[14] A. Farina, A. Russo, and F. Scannapieco, "Radar detection in coherent weibull clutter," IEEE Trans. Acoust. Speech. Sig. Proc., vol. 35, no. 6, pp. 893-895, June 1987.

[15] B. Picinbono, "Spherically invariant and compound Gaussian stochastic processes," IEEE Trans. Inform. Theory, pp. 77-79, Jan 1970.

[16] K. Yao, "A representation theorem and its applications to spherically invariant random processes," IEEE Trans. Inform. Theory, vol. 19, no. 5, pp. 600-608, Sept 1973.

[17] M. Rangaswamy, D. D. Weiner, and A. Ozturk, "Non-Gaussian vector identification using spherically invariant random processes," IEEE Trans. Aero. Elect. Sys., vol. 29, no. 1, pp. 111-124, Jan 1993.

[18] E. Conte, M. Lops, and G. Ricci, “Asymptotically optimum radar detection in compound-Gaussian clutter," IEEE Trans. Aero. Elect. Sys., vol. 31, no. 2, pp. 617-625, April 1995.

[19] F. Gini, "Sub-optimum coherent radar detection in a mixture of K-distributed and Gaussian clutter," IEE Proc. Radar Sonar Navig., vol. 144, no. 1, pp. 39-48, Feb 1997.

[20] E. Jay, J-P. Ovarlez, D. Declercq, and P. Duvaut, "BORD: Bayesian optimum radar detector," Sig. Proc., vol. 83, no. 6, pp. 1151-1162, June 2003.

[21] F. Gini and M. V. Greco, "Covariance matrix estimation for CFAR detection in correlated heavy tailed clutter," Sig. Proc., Special Section on Sig. Proc. with Heavy Tailed Distributions, vol. 82, no. 12, pp. 1847-1859, Dec 2002.

[22] E. Conte, A. De Maio, and G. Ricci, "Recursive estimation of the covariance matrix of a compound-Gaussian process and its application to adaptive CFAR detection,” IEEE Trans. Sig. Proc., vol. 50, no. 8, pp. 1908-1915, Aug 2002.

[23] F. Pascal, Y. Chitour, J-P. Ovarlez, P. Forster, and P. Larzabal, "Covariance structure maximum-likelihood estimates in compound Gaussian noise: Existence and algorithm analysis,” IEEE Trans. Sig. Proc., vol. 56, no. 1, pp. 34-48, Jan 2008.

[24] F. Pascal, P. Forster, J-P. Ovarlez, and P. Larzabal, "Performance analysis of covariance matrix estimates in impulsive noise," IEEE Trans. Sig. Proc., vol. 56, no. 6, pp. 2206-2217, June 2008.

[25] N. H. Lehmann, A. Haimovich, R. Blum, and L. Cimini, "MIMO-radar application to moving target detection in homogenous clutter," in Proc. Adaptive Sensor Array Processing Workshop, Lexington, USA, June 2006.

[26] Kraut S, Scharf L L, and L T McWhorter, “Adaptive subspace detectors," IEEE Trans. Sig. Proc., vol. 49, no. 1, Jan 2001.

[27] M. Abramowitz and I.A. Stegun, Handbook of Mathematical Functions, National Bureau of Standard AMS 55, 1st edition, June 1964.

[28] I. D. Olin, "Amplitude and temporal statistics of sea clutter," in IEE Conference Publication 216 for Radar, London, UK, Oct 1982 .

[29] D. B. Trizna, "Statistics of low grazing angle radar sea scatter for moderate and fully developed ocean waves," IEEE Trans. Ant. Prop., vol. 39, no. 12, pp. 1681-1690, Dec 1991.

[30] F. Gini, M. V. Greco, A. Farina, and P. Lombardo, "Optimum and mismatched detection against K-distributed plus Gaussian clutter," IEEE Trans. Aero. Elect. Sys., vol. 34, no. 3, pp. 860-876, July 1998.

[31] F. A. Pentini, A. Farina, and F. Zirilli, "Radar detection of targets located in a coherent K distributed clutter background," IEE Proc. F, vol. 139, no. 3, pp. 238-245, June 1992.

[32] F. Pascal, J-P. Ovarlez, P. Forster, and P. Larzabal, "On a SIRV-CFAR detector with radar experimentations in impulsive noise," in Proc. European Signal Processing Conference, Florence, Italy, Sept 2006.

[33] I. S. Reed, J. D. Mallett, and L. E. Brennan, "Rapid convergence rate in adaptive arrays," IEEE Trans. Aero. Elect. Sys., vol. 10, no. 6, pp. 853-863, Nov 1974. 
[34] F. C. Robey, D. R. Fuhrmann, E. J. Kelly, and R. Nitzberg, “A CFAR adaptive matched filter detector," IEEE Trans. Aero. Elect. Sys., vol. 28, no. 1, pp. 208-216, Jan 1992.

[35] E. J. Kelly, “An adaptive detection algorithm,” IEEE Trans. Aero. Elect. Sys., vol. 22, no. 1, March 1986. 